\title{
Original Article \\ Knowing as practice: Self-care in the case of chronic multi-morbidities
}

\author{
Susan Pickard ${ }^{\mathrm{a}, *}$ and Anne Rogers ${ }^{\mathrm{b}}$ \\ aSchool of Sociology, Social Policy and Criminology, University of Liverpool, \\ Room 1.13, Eleanor Rathbone Building, Bedford Street South, Liverpool L69 7ZA, UK. \\ E-mail: susan.pickard@liverpool.ac.uk \\ ${ }^{\mathrm{b}}$ NPCRDC, University of Manchester, Williamson Building, Oxford Road, Manchester \\ M13 9PL, UK. \\ E-mail: Anne.Rogers@manchester.ac.uk \\ *Corresponding author.
}

\begin{abstract}
Patient expertise in self-care is recognised as a cornerstone of disease management in advanced welfare capitalist societies. When conceptualised within a broader agenda of 'engaged and active patients' such expertise is expected to relieve UK NHS resources significantly. However, although self-care is first and foremost an embodied practice, grounded in the context of everyday life, state sponsored self-care support initiatives such as the Expert Patients Programme operate a dualistic framework separating cognitive and corporeal elements. Moreover, chronic disease management operates through a framework that is increasingly biomedical, specialised and reductionist. Patients with multiple morbidities in particular are not served well by this epistemological approach. Utilising a 'lived body' conceptual paradigm and drawing upon qualitative data gathered from interviews with patients with multi-morbidities, we explore embodied self-awareness in health and illness and the everyday practices of chronic illness work. We examine how patients integrate the different types of knowledge and practices resulting from interaction with primary care professionals and highlight the implications for primary care practice, for medical epistemology and for the democratic potentiality of the NHS.
\end{abstract}

Social Theory \& Health (2012) 10, 101-120. doi:10.1057/sth.2011.24;

published online 18 January 2012

Keywords: self-care; multi-morbidity; QOF; primary care; lived body

\section{Introduction and Aims: What Place for Lay Expertise in Chronic Disease Management (CDM)?}

Over the past decade a new policy focus on patient chronic illness and self-management has come to the fore as part of a care transition in which the

(C) 2012 Macmillan Publishers Ltd. 1477-8211 Social Theory \& Health Vol. 10, 2, 101-120 
official attention given to managing long-term conditions has begun to recognise an epidemiological transition (the prevalence and incidence in chronic conditions replacing acute conditions). State-sponsored self-management, including the Expert Patients Programme (EPP), forms a central part of this care transition, a policy that has placed patient action at the core of the work of participating with personal illness management on the one hand but also, on the other, of thereby helping the state to contain costs and implement a policy of demand management (DH, 2005). One way in which policy has sought to develop patients as experts is through the increased involvement of active and informed patients in health-care decision-making and self-management in primary care (Calnan and Gabe, 2009).

However, although self-care is above all an embodied practice, grounded in the context of everyday life, which includes the support of lay care-givers in a context of interdependency, both CDM support and self-care in primary care consultations approach patients through a dualistic framework separating mental and bodily elements. Thus EPP valorises cognitive practices, focusing on improving self-efficacy and imparting general coping strategies, better breathing and healthy eating, improved communication and working with health-care professionals (Rogers, 2009). As such, its patient model resembles that of 'Rational Man' (sic) (Young, 1981) privileging objective, logical and autonomous decision-making. In addition it is intended to inculcate an ideal typical late-modern patient: responsible, self-directed and managing her own health (ref removed). This, it is hoped, will remove the need for secondary care and thus support the continued financial viability of the NHS (Wanless, 2002).

Meanwhile, the structures and processes through which CDM is delivered in primary care are, following the introduction of the Quality and Outcomes Framework (QOF), increasingly standardised and bureaucratised around adherence to specific measurements of bodily normality derived from epidemiological evidence and applied to single diseases in isolation from others that may be experienced simultaneously by patients (Checkland et al, 2008). Indeed, the coexistence of conditions is very common: individuals living with multiple long-term conditions, or multi-morbidities, currently make up 45 per cent of the total number of approximately 17.5 million adults in the United Kingdom living with chronic illness (DH, 2008). Although there is limited ethnographic data describing self-care in the context of multi-morbidities, and particularly data detailing the interplay between embodied practices of self-care and primary care interventions, we know that, as well as presenting a challenge to clinical care, self-care in the context of multi-morbidities is more challenging than in the context of single conditions (Bayliss et al, 2003). 
Further exploration of lay expertise in this context, we suggest, will thus serve two purposes. Epistemologically, there is a requirement to understand the nature of lay expertise, how it is acquired and applied over the course of the illness trajectory in the context of multi-morbidity, and how it works with other kinds of knowledge, particularly biomedical knowledge. This would, secondly, permit the design of appropriate and sensitive professional support for lay self-care in this context. Our aims in this article are underpinned by sympathy with previous research (for example Sullivan, 1986; Mol and Law, 2004), which points to the necessity of transforming the epistemological as well as organisational foundations of contemporary CDM to acknowledge the validity of experiential knowledge and action based on practice grounded in everyday life.

The article aims to make a contribution towards this objective by utilising qualitative data obtained via patient interviews. First, it will set out the conceptual framework through which we have chosen to analyse our data, namely that of the 'lived body'. We will then describe, drawing on one detailed case study, the practice of embodied self-awareness in health and illness and the experiential knowledge and embodied processes (cognitive, corporeal and social), which together form the foundation of 'chronic illness work' (Corbin and Strauss, 1985). Next, we will set out the contrasting logic and practice that underpins CDM in primary care before going on to consider how the patient integrates these two different knowledges into her self-care. The concluding sections will highlight the implications of the findings for primary care practice, for medical epistemology and, more generally, for the democratic potentiality of involving patients and citizens in health-care decision-making.

Ahead of this, however, a theoretical preamble will set out why we chose to pursue our exploration of self-care through the lived body paradigm in preference to alternative paradigms within the sociology of the body.

\section{Bodies of Knowledge, Knowledge of Bodies}

There are, broadly, two main perspectives in which sociological approaches to the body can be situated. These are: (i) accounts that privilege social structure, the degree to which body-as-object is inscribed by political and social discourses from the outside: 'what is done to the body'; and (ii) those that focus on agency: the experience of the body-subject within her social world, or 'what the body does' (Crossley, 1995, 1996; Howson and Inglis, 2001). Although this distinction (again, broadly) adheres to the emic/etic divide, discrete perspectives may be more or less dualistic in their approach to the body, in a way that does not divide neatly between the two categories. 
Meanwhile, in terms of sociological research into the experience of people living with chronic illness, we can identity three dominant traditions: (i) social constructionist, (ii) narrative identity and (iii) lived body paradigms. Social constructionism approaches the illness experience of bodies in terms of history (genealogy) and politics, critically examining the role of medicine in constructing discursive categories of sickness and disease, defining what is 'normal' for a body and shaping the subjectivities of patients accordingly. Although the body is a key site for the operation of power in this paradigm, it can resemble an object with very little attention given either to its fleshy characteristics or to the experiential dimension (for example Turner, 2001). Second, narrative approaches look at the experience of illness and disability through a focus, derived from Mead, on the social shaping of self and identity. Although this usefully blends social, cultural and individual elements it has tended to adopt a predominantly cognitive view of the self with more emphasis accorded to the way the self restores biographical meaning and adjusts to changes in identity following impairment or other changes to the body (Kelly and Field, 1996). Finally, the lived body approach as it has developed in the humanities and social sciences, drawing on the phenomenology of Merleau-Ponty in particular, focuses on the corporeal experience of illness and disability within the lifeworld, highlighting experiential aspects such as changes to embodied self-awareness, motility, spatiality and temporality. Its limitations are those Bourdieu (2000) identified and sought to remedy through the theory of the habitus, specifically its neglect in theoretical terms of the influence of socio-structural factors on embodied dispositions (a neglect deriving from its philosophical roots).

Although all three paradigms provide helpful insights through which to approach the questions we set ourselves - the nature of lay expertise in self-care - we chose to utilise the lived body perspective as most apposite. More recently applied to bodies in health and illness by contemporary philosophers such as Drew Leder (1990), S.K. Toombs (1992) and others, its utility for the sociology of the body has been richly demonstrated by sociologists such as Simon Williams, Gillian Bendelow (Williams, 1996; Williams and Bendelow, 1998) and Nick Crossley (1995, 1996, 2001), who have also demonstrated its possibilities as a method to explain situated experience (see also Young, 2005). In terms of understanding the expertise of self-care in particular, this utility has several dimensions. First, it redresses the emphasis given to cognitive processes and puts the body firmly back into the centre of the account. Second it attends to the experience - the 'chronically ill body', 'self-care' - that is social constructed (Turner, 2001). Third, and perhaps most importantly, it adopts a non-dualistic approach to knowledge that convincingly challenges: the primacy of critical reflection; the notion of an 'inner mind' relating to an independent 
outer object; the idea that action is underpinned by (explicit or implicit) theorising; and the superiority attached to objectivity, autonomy and detachment towards the social world (see Dreyfus, 1991). The lived body paradigm suggests that our experience of everyday embodied practices is itself a fact about the world, compatible with genuine scientific enquiry, and indeed without which understanding biomedicine remains phenomenologically inadequate (Carman, 2008).

Although in recent years the lived body perspective has contributed to the project of rendering the sociology of chronic illness more embodied, we note a parallel trend in the sociology of disability that is reintroducing impairment into the disability debate (Williams, 2006). Although previously discussions of the experience of disability were framed predominantly within the terms of the social model of disability, a Cartesian model similar to that found in biomedicine has resulted where, as Hughes and Paterson point out, 'both treat [the body] as a pre-social, inert, physical object, as discrete, palpable and separate from the self' (1997, p. 329). Thus, an understanding of the phenomenological parameters of self-care is as relevant and important, by this reading, to sociological understanding of disability and impairment (and in certain circumstances ageing too), as it is to the understanding of chronic illness.

We discuss these parameters next.

\section{The Lived Body: Embodied Self-awareness of Health and Illness}

With his rejection of Descartes' approach to perception, Merleau-Ponty posits embodiment as the basis of experience and perception as the grounds of subjectivity, with the primary function of perception being not contemplation but practical action (Crossley, 1995). To understand is thus an act, not a cognitive contemplation, consisting in competent bodily action 'whether that be the action of applying a learned mathematical formula or of driving a car' (Crossley, 1995, p. 55). Two key aspects of the body's role in perception are identified by Merleau-Ponty (1962) as the 'body schema' and 'motor intentionality'. The former suggests a dynamic ability to engage with the world in terms of postures and bodily behaviours that make acts possible: the body is 'polarized by its tasks', it exists 'towards them' (Merleau-Ponty, 1962, p. 115) via the body schema. Motor intentionality, on the other hand, refers to pre-reflective, skilful physical behaviour: a non-cognitive intelligent bodily awareness of self and environment (Carman, 2008). The combination of both in practical knowledge, or 'praktognosia', is pre-linguistic and habitual. Above all 'the world is not what I think but what I live through’ (Merleau-Ponty, 1962, p. xviiii). Intentionality, meaning and experience when sufficiently attuned can facilitate one's 'best grip 
on the world' (Merleau-Ponty, 1962), a set of bodily attitudes that enable us to place ourselves within the world most harmoniously.

Somewhat ironically, the lived body also demonstrates the experiential basis for the persistence of dualism in western consciousness. Indeed, an element of bodily self-awareness is the experience of oneself both as subject and at times as object thus actually seemingly reaffirming the dualist approach favoured by medicine (Leder, 1990). For example, although we may be aware of particular outer elements of the body when using them - such as hands or feet - this requires the enlistment of the rest of the body in a quiescent and supportive 'background attitude', summed up in the notion of figure-ground gestalt. One can then talk about a 'phenomenological anatomy' of the lived body in terms of the usual habits of presence and absence, the activities they entail and the world-relations associated with them (Leder, 1990). In this schema, the inner organs lie mostly beyond conscious perception. However, at the same time they do participate in our experiential state and constitute part of one undivided bodily intentionality so that, for example we experience fatigue in multi-layered terms as a combination of metabolic processes in the muscles, changes in the chemical composition in the blood, hormonal shifts and mood or mental disturbance, which in combination effect a qualitative transformation of one's entire being-in-the-world (Leder, 1990).

Illness disrupts the usual phenomenological anatomy. One becomes conscious of one's body or part(s) of one's body in a way that Leder terms 'dys-appearance' (defined as the appearance of the body in a 'dys' - bad, hard, ill - state) and which disrupts practices, roles and relationships with others in everyday life. This then requires the imposition of some new coherence in terms of biographical continuity and coping strategies. Transformations in bodily awareness are particularly durable in chronic illness, which may be experienced as a prolonged, if fluctuating, set of tensions that frustrates the familiar features of embodiment, constricting one's sense of space, transforming temporality, impeding bodily intentionality, and thence self and world (Toombs, 1992). In multi-morbidity, these fluctuations and the change in gestalt-patterns associated with them, will certainly be all the more complex and nuanced.

Williams (1996) has helpfully synthesised the corporeal and cognitive dimensions of this experience in a more sociologically focused explanatory framework as follows. Illness leads to a state of 'dys-embodiment' or the 'resurrection of dualism', wherein bodies become negatively conscious of themselves and taken-for-granted relationships between body, self and society become problematised. Following struggles at all these levels of experience, individuals may succeed in realigning mind and body, self and society, through a range of biographical and practical adjustments leading to the restoration 
of a sense of continuity or normality (Bury, 1982). This leads to a new 're-embodiment', in terms of a non-dualistic body-self albeit one that is achieved, and is the product of work, rather than something taken-for-granted; all the more so in that, in chronic illness, this achievement is frequently unstable and may require further ongoing work. Thus the chronically ill person learns to juggle an ultimately irresolvable set of tensions over which s/he must impose some sense of coherence, if re-embodiment is to be maintained (Corbin and Strauss, 1985). In this process, patients may choose to preserve aspects of their identity or habitus at the cost of symptom management. An obvious example is where the individual may resist using a wheelchair, despite significant impairment, because of its symbolic character that is suggestive of disability and thus threatening both to self-perception and social identity (for example Toombs, 1992). As Turner expresses it, 'Embodiment and enselfment are mutually dependent, interdependent and reinforcing projects' (2001, p. 252).

One important aspect of chronic illness work is the necessity to integrate self-care with interactions with professionals, involving the incorporation of medical regimens based on a radically different approach towards the meaning of chronic illness together with a contrasting knowledge of the body. We consider this next.

\section{Epistemologies of the Body in Primary Care}

As we noted earlier, it has been observed that CDM within primary care is increasingly situated within a biomedical framework wherein symptom management within 'normal' values is the dominant or exclusive priority (Checkland et al, 2008). CDM in UK general practice is driven by a 'pay for performance' scheme called the QOF introduced by the 2004 General Medical Services General Practitioner (GP) contract, which utilises standardised protocols for the management of specific common chronic diseases. These are predominantly clinically focused: for example, the (2010) QOF for diabetes gives a required range of normality for readings of blood pressure and HbA1c and also categorises the status of the foot of the diabetic into several discrete categories according to the presence of morbidity. These protocols are applied to individual patients by means of annual disease reviews, which are initiated by standard call and recall letters to patients classified as falling into the relevant (single) disease categories. They are administered by nurses who increasingly specialise in these discrete areas, overseen by GPs who lead in these disease areas and who carry out the annual medication review (Checkland et al, 2008).

This protocol-based approach, privileging evidence-based medicine, has been described as inevitably limiting the ability of patients to introduce their own 
priorities (for example Rhodes et al, 2006). It also restricts GPs' abilities to engage with the lived experience of multi-morbidity for a variety of reasons, relating both to the nature of evidence and to the structure of doctor-patient interaction contained within the framework. Not only does the 'evidence' base of the protocol privilege the methodology of probabilistic sampling of large numbers over the particularly of individual bodies (Byrne, 2004) but the layout of the templates associated with each specific disease framework leaves the GP little or no room to record data, including social or biographical context or, indeed, any medical impressions that are difficult to quantify (Berg, 1997). As Blaxter (2009) observes, although it may no longer be entirely accurate - owing to changes in medical education as well as shifts in patient attitudes - to oppose the 'world of biomedicine' to the 'lifeworld', in this context the significant opposition may be the qualitative views of the patient/doctor versus the 'evidence'. Furthermore, increasing specialisation inevitability fragments continuity of care, which will further compromise clinicians' engagement with the patients' lifeworlds.

In approaching the body in narrowly clinical terms, the framework does not take account of the fact that, for example in the case of diabetes selfmanagement, deciding to aim for a higher or lower blood sugar level is not simply a clinical but also an existential choice as to, for example, whether one would prefer to risk blindness or kidney failure in future years for the purpose of avoiding comas that make it dangerous to drive in the short term (Mol and Law, 2004). In multi-morbidity, prioritising treatment for one particular condition may have positive effects, for example, on one's social life but, simultaneously, negative effects on other bodily conditions. Thus, the CDM version of 'health' as something assessed through measurement contrasts with that of many lay people who perceive health in a way that bridges the physiological and social, such as the ability to do the things they want to do (Blaxter, 2004). It is also possible that in the case of multi-morbidities the aim of achieving a certain 'number' for one condition may disrupt the gestalt flow in which patients have learned to self-manage the whole and thus may in practice introduce instability or deterioration.

\section{Methodology}

The following data derive from qualitative research that examined CDM processes in primary care for patients with multi-morbidities. Following ethical approval, patients who lived with two or more chronic conditions (left undefined) were selected by practice managers randomly from their lists and letters of invitation were sent out explaining the research. Four patients in each 
practice who gave their formal consent were interviewed up to five times each over the course of a year by one researcher, for consistency. Carers were interviewed where appropriate because, against the model of the 'autonomous, rational patient' found in policy documents, we anticipated that self-care would involve a strong element of intersubjectivity and interdependence, including family and familiars. The longitudinal approach aimed to chart changes over time, in terms of the interaction between health, life events and contacts with health-care providers in primary and secondary care and to capture as far as possible 'in real time' patients' varying methods for self-managing.

Questions at initial meetings aimed to explore the conditions with which patients had been diagnosed, the history of the illness trajectory including contact with health-care professionals and self-management techniques. Subsequent interviews explored recent changes in the stability of one or more conditions as well as returning, in greater depth, to aspects covered in the first interview. This was particularly helpful as patients' tacit knowledge required time and patience to draw to the surface and could not always be elicited through direct questioning.

We have elected to present data in the form of one case study, that of 'Rita', (not her real name), a patient in 'Treetops' practice in a deprived inner-city area of Greater Manchester. Although focusing on this one case sacrifices breadth in favour of depth, this approach was chosen because of the opportunity to provide rich detail at a micro-level, the better to reveal the interplay between different kinds of practices and different types of knowledge. Rita had been diagnosed with a wide range of chronic illnesses of many years standing, associated with an original diagnosis of diabetes, and including coronary artery disease, moderately advanced chronic kidney disease (CKD), hypertension, osteoporosis, neuropathy and gout. She has contact with a wide range of professionals in varied settings and receives annual and interim disease-specific reviews at her general practice for the diabetes and CKD, respectively, arranges frequent ad hoc appointments at the practice with a range of GPs and nurses, and in addition attends outpatient clinics at two different hospitals for diabetes and CKD, respectively. She had not been offered any opportunity to attend self-management training programmes. She takes approximately 12 different medications daily.

The data will be presented in terms of three themes. First, focusing on embodied self-awareness, we will examine the interrelationship of Rita's various morbidities in corporeal terms, how they fit together in combinations that are complementary, neutral or antagonistic and how bodily knowledge in this context includes ability to read and interpret signs relating to different origins or causations. Second, we will examine the situated dimensions of her chronic illness work, including maintaining multiple regimens, medicines and 
therapeutics in the context of everyday and valued practices, roles and relationships and the interplay between them. Third, we will look at the role played by health-care professionals and the place of biomedical regimens in supporting this illness work.

\section{Rita: Contextual Background}

Rita, an East-African born former nurse of Indian heritage, is in her early fifties, married with one grown up son who still lives at home. She developed insulindependent diabetes as a child and during the past 15 years the additional conditions noted above also emerged. Her tiny modern terrace home was in a treeless housing estate in a post-industrial socio-economically deprived area and she possessed considerably more cultural/educational than financial capital.

In terms of her contact with clinicians at Treetops, the GP she sees most frequently is Dr Whippet, the senior partner, who leads on CKD and with whom she has a relationship extending back many years. He is a 'traditional' GP who has been at the practice some 30 years and prides himself in living in the area and consciously continuing the heritage of the practice back to its original founder at the turn of the previous century. While she sees him for her regular CKD review, Rita also attends frequently for ad hoc appointments (up to six times a month); if she is unable to secure a timely appointment with him then, as second choice, she sees Dr Samuels, a younger partner who has been at the practice some 10 years and who leads on stroke (and thus does not administer her disease review). She sees Dr Clarke, a part-time female practitioner, for the diabetes review only. Outside of this, her disease has progressed to the extent that she also sees secondary care clinicians based at two clinics at two different hospitals, with a general physician and renal specialist team, respectively, at approximately six monthly intervals. Dr Whippet reviews any new medication prescribed for Rita by her hospital specialists.

One of us SP interviewed Rita at her home on three separate occasions: the first time, osteoporosis and gout were particularly troubling her; on the second occasion, her kidney disease was prominent, owing to the effect of receiving news regarding a sharp deterioration in function; on the third the kidney function remained foregrounded, following a discussion in which she was informed of the likelihood of needing kidney transplantation. During the latter part of this last meeting Rita's 26 year-old son joined us and discussed his own role in giving care and supporting his mother's self-care. A further two telephone conversations took place subsequent to the last interviews for the purpose of clarifying certain details in the material. 


\section{Embodied Self-awareness in the Context of Multi-morbidity}

During the vicissitudes of her illness, Rita had gone from monitoring her blood sugar to managing the consequences of multi-morbidities associated with several other organs. She describes the daily experience as 'Like a juggling act ...' and elaborates:

You start off, have I got any swelling on my feet, then come down and you think, right let's check the blood sugars and see what they're like. My bones are worse in the morning ... and then it's the thing how you progress through the day.

Rita illustrates how embodied self-awareness, although built on a preobjective relationship with our bodies and the world, is suffused with meaning that is necessarily social and cultural. Thus, she has learnt to make consideration of her blood sugar needs of primary salience in her self-awareness, although it is now tacit. These needs are, she describes, 'a foundation ... (in) an interlinked chain'. All conditions arise from the diabetes, explicitly or implicitly, but each has an effect on the whole: 'If one of them is weak, it starts, sort of, breaking'. Any condition arising outside of this has the potential to adversely affect it: for example, a cold or infection raises her blood sugar level significantly with effects lasting between 4 and 6 weeks. Moreover, the fluctuations of sensations localisable in body parts and organs located at the surface of the body - temples, ears, feet and so on - present clues to the status of underlying conditions located in the recessive inner body: that her gout was no longer troubling her indicated to her that her kidneys were stabilising.

Elements of Rita's 'interlinked chain' variously surge to the forefront or ebb to the background in a constantly shifting dance. There are a variety of reasons why one condition will assume prominence. Some arise from the body itself, as in the case of pain: when experiencing the severe osteoporosis-related backache to which she is prone everything else recedes. Others arise from interaction with health-care providers, such as receiving test results: her kidney disease was in the 'back of her mind' until test results indicated reduced function: 'So then this osteoporosis thing gets to the back and then you bring the kidney things in the front'. Other reasons stem from her personal biography: so for example, diabetes, normally her key priority, will recede from her attention when valued activities demand it. One of the examples Rita gives is that of pilgrimages to Mecca, which she has carried out three times. In each case, her blood sugar level went 'through the roof' but for once this did not matter to Rita as long as she made it through each day.

Through constant surveillance of her body Rita has learnt to interpret phenomenological states linked with multi-morbidities in a sort of hermeneutic 
circle wherein surface and recessive aspects of the body form one variegated but unified existential state or condition. She describes, for example, four distinct types of fatigue each linked with different 'silent' organs or body systems. With CKD, 'you're sleeping a lot, that sort of tiredness'. With getting over a cold, 'it's tired, a bit like weary-tired'. When her blood sugars are high, it's more like lethargy: 'You don't want to get up. You don't want to do anything'. Alternatively when they are very low: 'You float away into this sleep, and you're like you're on air'. Similarly there are three contrasting types of headache: a hyperglycaemic headache is very severe and 'you can actually feel your arteries pumping'. A hypoglycaemic headache is 'like a hangover and your eyes feel heavy'. A common-and-garden headache is different again: a tight band at temples and neck. Leg pain too can be differentiated according to its origin in either high blood sugar levels, neuropathy or gout. These responses are unique and cannot thus be captured either in medical textbooks or self-care manuals. Such knowledge enables Rita to detect subtle changes to the norm, although they may then require further 'detective work': 'Your feet don't swell up just because there's something wrong with your kidneys, it could be anything'. Sometimes the complexity of this task may lead to her seeking professional interpretation, although the hermeneutic element involved normally requires Rita's continued input. For example, she noticed an elevation in her hypertension as follows: 'When you lie down, you can hear "bang, bang, bang” or you can hear like a hissing sound'. The senior GP partner, Dr Whippet, suggested it was a problem with her ear drum:

And I kept thinking there is nothing wrong with my ears, I know it's not my ears. Anyway ... they introduced another new heart medication and then one night I suddenly thought, hmm ... . It's gone.

This is an example of Rita being not just self-aware - the non-thematic proprioception (or body's awareness of itself) of Merleau-Ponty's phenomenology - but also aware of the effects of medical interventions on her body (Mol and Law, 2004). However, despite her dense experiential knowledge, her body remains, intractably, unpredictable, unlike that of a machine, and is never fully 'mastered' (as ultimately no body can be); sometimes her blood sugar levels fluctuate for no obvious reason, despite eating the same things every day and injecting the same dosage of insulin, perhaps because she has forgotten to take into account 'small things' like a walk earlier in the day or the weather, or perhaps for no reason at all. Sometimes, it is her son who notices changes from Rita's norm (thereby highlighting the importance of knowledge as continuity in this context) and takes remedial action: 'I can be sat here and my blood sugar level can be 2.5 and getting lower and I'll be fine but I don't recognize the signs, Raj is the one who recognizes the signs.' Raj also knows 
how to 'distinguish' chest pains relating to muscular problems or side effects of medication from angina, according to her mode of breathing, her general demeanour and her pallor, in a way that supplements, reinforces or even (in the case of a hypo) contradicts Rita's self-knowledge. At other times, her embodied self-awareness may prove to be out of alignment with biological events and thus contradicted by test results. Thus she has 'no idea' what the kidney function reading will reveal when she attends her outpatients' appointment at the renal unit; levels of creatinine or urea in the blood or protein in the urine are beyond her perceptual grasp until and unless they produce signs affecting urination, back pain or fatigue, and then only indirectly, and by way of influencing perceptual states such as fatigue or headache or affecting parts of the body furnished with more nerve endings and receptors such as in the case of gout. Finally, there are times when demands ensuing from one part of her body means that she attends to this without considering the consequences for the rest, as during a recent episode of severe backpain when she took painkillers and 'totally forgot' about its detrimental effect on her kidneys.

\section{Self-care as Effecting and Maintaining Re-embodiment}

Re-embodiment for Rita involves constant work. Medication for any condition can raise her blood sugar level, which is then a struggle to normalise. Rita chooses to maintain her blood sugar level at the lower end of the normal range, despite the risk of hypos, including most dangerously in the night, as well as the concerns of her family but, in discussion, reveals an embodied logic for choosing to risk hypoglycaemia over other possibilities for her body. First, she believes that higher blood sugar is a 'slow time-bomb ... tick-tick-tick', risking almost certain damage to eyes, kidney and nervous system. Second, her body 'agrees' with lower blood sugar in other ways: not only can she function normally most of the time at a very low level but hypoglycaemia is associated for her with 'pleasant' feelings 'a bit like being high'. By contrast, her blood sugar level has only to creep up to an average of $7.6 \mathrm{mmol} / \mathrm{l}$ - not particularly high by clinical standards - for her to start to feel 'thirsty and ill'. There is a third, and key, factor that facilitates this choice, namely her son's presence in the home, whom she describes as 'properly trained' to manage a hypo should it occur.

Rita's use of medical technology facilitates her bodily self-knowledge. She describes herself as being 'on the spot 80 per cent of the time' in terms of her own awareness of her blood sugar, but symptoms may be masked in the remaining cases as a result of the interactions with other morbidities. For example, frequent urination, a once-reliable sign of low blood sugar, is also now associated with medication for CKD. Its source can, fortunately, be clarified by using her blood testing kit. Technology has also enabled her to maintain 
valued roles, practices and identity. Thus, a change in diabetes medication several years before - to a longer lasting type no longer requiring refrigeration meant that she was able to perform the Hajj (the annual Moslem pilgrimage to Mecca and Medina in Saudia Arabia already noted) for the first time. She has subsequently gone on the Hajj on three occasions over the past 8 years (on one occasion discharging herself against her doctors' advice from a cardiac ward in order to make her flight). Despite the very high blood sugar readings - up to $20 \mathrm{mmol} / \mathrm{l}$ at times - experienced during the trip, she believes it to have had a very positive effect on her in that it restored to her a sense of her body's possibilities, which for her is one meaning of health itself: 'You think, I've got these problems but yes, I can do this. So that means I can't be that bad. It helps you cope'. This has a broader role in facilitating health as her religious faith has sustained her in both cognitive and practical ways. Cognitively, it provides both meaning and a way of coping, while sustaining biographical continuity. She says that it is her conviction that 'if (God) has given you this problem, then $\mathrm{He}$ is the one that is going to help you cope with it'. Practically, she explains that keeping Ramadan has helped inculcate in her the ascetic dispositions required by diabetes self-management as, while the fasting associated with it is not beneficial in the short term, causing the blood sugar to rise alarmingly, nevertheless in terms of more permanent dispositions beneficial to self-care, it 'teaches you a lot of self discipline, eating and drinking'.

Diet and nutrition provide an excellent example of the difficulties in self-care where tensions may coexist within and between her biological body, her roles and her relationships. Therefore although she had modified her diet many years ago in accordance with her diabetes, when coronary artery disease was responsible for a heart attack requiring two angioplasties she was obliged to make further changes by checking not just sugar and carbohydrates but fat content also. This necessitated preparing separate meals for herself and for her family on a daily basis. It also set her apart from friends and family during celebrations and communal meals:

Yes, because you see while everybody is at the table [putting food] on their plate, you're weighing up, you're stood back weighing up how much of this can I have and how much of that. And they would say, 'What are you thinking? Get the food on your plate'.

One complicating factor for Rita is that although certain foods are promoted for their health-giving properties, they may not serve all her conditions equally well. So, she explains,

If you look at the heart situation it says eat lots of fruit, eat lots of vegetables ... but the fruit is sugary so you can't just eat fruit and vegetables .... So although it is good for you, in a diabetes sense it is not. 


\section{The Role of Health-care Professionals in (Re)embodiment}

The frequency of Rita's attendance at 'Treetops' means that she has regular contact with at least two of her GPs outside disease reviews and sees a third GP exclusively for disease reviews. As we have noted, she also sees two hospital specialists (or specialist teams) This then presents an opportunity to compare differing approaches to supporting her self-management, associated with different styles of interaction and different types of knowledge.

Dr Whippet is key to Rita's successful self-management and one way in which she describes his central role (which is also, to a lesser extent, played by $\mathrm{Dr}$ Samuels) is in terms of his appreciation of the instability of the re-embodiment she has painstakingly achieved and a reluctance to upset it by introducing new medication. So Dr Whippet will not prescribe medication 'unless it is absolutely necessary' and Dr Samuels 'prescribed' her Jewish chicken soup instead of antibiotics for a heavy cold. She calls this approach taking 'a step back to look at the broader view of things', a cautious approach comparable to her own.

She compares this favourably to the more reductionist approach of the third GP, Dr Clarke, whom she mainly sees within the context of disease reviews and of her general hospital physician, Dr Barot, and which Rita calls 'tunnel vision'. The 'tunnel vision' physicians are also more inclined to suggest pharmaceutical intervention, their focus being on the (single) disease rather than the (broader) implications in terms of her other illnesses and their interplay. For example, Dr Barot prescribed steroids for pain because he felt that they would 'only' raise her blood sugar level 'for four or five days' - unaware of the delicacy of her re-embodiment and its contrasting experience of temporality. She relates angrily, 'And I was thinking, to you it might only be four or five days but for me, it's going to take me two to three weeks to bring it back to normal!'

As noted, she relies upon her GPs to carry out tests to investigate signs and symptoms or confirm any suspicions she might entertain. A pain in her knee initially diagnosed as osteoporosis was subsequently discovered to be gout; pain in her back initially suspected to be kidney pain was discovered following an MRI scan to be osteoporosis. Sometimes Rita requests specific tests, as when she 'knew' that a pain in her chest required an angiograph as it was 'not muscular pain' and Dr Whippet expressed his confidence in her judgement. These examples illustrate the importance of lay and professional interpretive logic proceeding in partnership in solving these ongoing puzzles, and of valuing patients' experiential knowledge as equally important as their own.

The contribution that Rita values most highly from her clinicians is their knowledge of her as a 'person' not just a patient, including both her clinical and personal history, which enables them to tailor their advice so that it is 
appropriate to her everyday activities as well as biography. As we have already noted in the case of her son's observation, understanding of her normal stance of being-in-the-world is crucial for an understanding of morbid changes to that. Dr Samuels, for example, will regularly begin a consultation with questions about her family, knowing that Raj in particular is a key factor in Rita's self-care. Dr Whippet has been similarly supportive of her performing the Hajj; rather than warning her against possible ill effects he supplied her with antibiotics and other medication to self-medicate should she need it while there, saying 'my job is to support you'.

By contrast, appointments at the hospital, often with doctors she has never met before within the renal team, generate great anxiety. During her most recent appointment, the new specialist informed her that she would need to lose weight:

I said, 'Why? My diabetes is in control! ... . I don't want to be Kate Moss!'And he said, 'To get onto kidney transplant, you have to be three and a half stone lighter for you to be even considered.' And I'm saying, 'Oh, right, okay.' Well, when I came home I couldn't stop crying, and I'm thinking, oh, nobody's ever said to me, you know ... because I've never looked that far ahead ...

She immediately made an appointment to see Dr Whippet. She recalls: 'Dr Whippet ... he said, “I wouldn't worry about it, it's okay, you just carry on what you're doing .... . At the moment all you need to concentrate on is to make sure that your diabetes is well controlled."' This response supported her self-care, clinically and existentially in that it contained her fears and directed her attention positively to tangible activities within her control and so helped her to re-establish her 'best grip' on the world.

\section{Discussion and Concluding Remarks}

In this article we have argued for an understanding of the more grounded and embodied aspects of living with multiple chronic conditions and an appreciation of the particular type of knowledge underpinning it. Utilising a lived body perspective, and drawing on the phenomenological approach associated with Merleau-Ponty in particular, we have been able to demonstrate that self-care employs an embodied, practical knowledge that is very different from the abstract, rational model of patient knowledge assumed in programmes like EPP. In lived reality, our patient demonstrates that knowledge is not something you have or acquire but something you do, and in a social, biographical and clinical context, that contains objects and other people as well as one's own 
body. An understanding of the practice of embodied self-care, we suggest, is key to effective professional support and may serve as a starting point from which to question CDM's focus on clinical indicators derived from epidemiological evidence and applied to single diseases. Of course, the call to integrate patients' lived experience into medicine is hardly a new one but such calls have mostly suggested adding this perspective to the clinical method (Sullivan, 1986) in a way that resembles the biopsychosocial approach and also risks its limitations (Armstrong, 1995; Mol and Law, 2004). From our data we would instead concur with Mol and Law's (2004) conclusion that, building from the epistemological starting point of knowledge as practical activity,

medicine should come to recognize that what it has to offer is not a knowledge of isolated bodies but a range of diagnostic and therapeutic interventions into lived bodies, and thus into people's daily lives. (2004, p. 58)

In fact, several physicians in our study do appear to understand this; often, however, they find themselves at odds with a protocol-based system that is increasingly biomedical and reductionist. Indeed, we do not wish to set lay and medical knowledge against one another nor to accord one superiority over the other, in whichever direction. That being so, we cannot concur with arguments (for example Prior, 2003) that point out the limitations on lay 'expertise' in terms of their inadequate understanding of technical knowledge such as disease causation and management. We do not accept uncritically biomedicine's definition of expertise and evidence, nor the implicit dualist ontology hidden within it, but rather resist their reification as social facts. Not to challenge such assumptions amounts to locking sociology into the structures of the 'governing mode' that perpetuate this power/knowledge nexus (for example Smith, 1974). In the words of Bourdieu, social science can unmask the strategies of the dominant because its goal is 'not only knowledge of an object ... but knowledge of the knowledge - practical and scientific - of a given object of knowledge' (2000, p. 83). The lived body approach, we hope we have shown, provides a keen conceptual means to execute this challenge as it demonstrates clearly that self-care expertise lies not in a technology or rational understanding but simply in the embodied and practical act of doing self-care. To paraphrase Merleau-Ponty, expertise in self-care just is self-care as practised.

In our case study, two of Rita's GPs understood that for Rita successful management of diabetes and the co-morbidities associated with it was about far more than achieving a standard target physiological reading. Rather, it was about integrating bodily management into life strategies, and thus neither took predominance over the others but all contributed to a sustained 
re-embodiment. Thus, blood sugar levels were consistent with Rita's life choices supported by the actions of her son and the sustenance of her religious beliefs. This also necessitated that at times glycaemic control had to be sacrificed for these other valued practices. Together, these bodily and social elements all form part of Rita's phenomenological anatomy and it is to this, not the textbook version of anatomy, that medicine contributes. At the same time, clinical techniques can be accommodated fully into such practical knowledge in the understanding that, as we noted from the experience of 'dys-appearance', objectivity is a 'normal' aspect of bodily experience, just as much as subjectivity (Jackson, 1996), although not one that should be deemed more legitimate.

This accommodation, we suggest, is difficult if not impossible to achieve with formal QOF-led CDM practices as they currently exist. Their concentration on single diseases, and the ever-increasing specialisation and bureaucratisation witnessed in primary care and other branches of medicine, points to a tendency in human consciousness to impose order and classify experience into clear categories, where multi-morbidities, with all their ambiguity, threaten to disrupt such order. Where such ambiguity can be accommodated in GP-patient interactions outside standard reviews, general practice remains most receptive of all branches of medicine to incorporating the lay perspective, inasmuch as GPs 'are faced with the challenges of providing bridges between the uncertainties of clinical practice and biomedicine's claims to epistemological privilege' (Busby et al, 1997, p. 94). The concern then is how GPs can integrate the standardised demands of QOF with the individualised requirements of their patients. Finally, we also suggest that the valorisation of knowledge as practice within medicine's epistemology would also play an important role in terms of wider processes of democracy in the NHS. Hierarchical structures and processes are, after all, intrinsically linked with the dualism in which the biomedical tradition is rooted and which has historically been associated with control through the construction of order that once 'discovered' through rational thinking can then be imposed upon others (Jackson, 1996). By contrast, knowing as practice supports subalternity, which suggests a refusal to privilege detached intellectual activity over the rich and complex expertise that is emergent directly from the lifeworld.

\section{About the Authors}

Susan Pickard is Senior Lecturer in Sociology at the University of Liverpool. She is writing a book that argues for a new and positive ontology of the ageing body departing from metaphors of decline and illness. 
Anne Rogers is Professor of the Sociology of Health Care at University of Manchester. She is currently developing theoretical approaches to the role of health services in supporting self-care of long term conditions.

\section{References}

Armstrong, D. (1995) Theoretical tensions in biopsychosocial medicine. Social Science and Medicine 25(11): 1213-1218.

Bayliss, E., Steiner, J., Fernald, D., Crane, L. and Main, D. (2003) Descriptions of barriers to self-care by persons with comorbid chronic diseases. Annals of Family Medicine 1(1): 15-21.

Blaxter, M. (2004) Health. Cambridge: Polity.

Blaxter, M. (2009) The case of the vanishing patient? Image and experience. Sociology of Health and Illness 31(5): 762-778.

Bury, M. (1982) Chronic illness as biographical disruption. Sociology of Health and Illness 4(2): 167-182.

Busby, H., Williams, G. and Rogers, A. (1997) Bodies of knowledge: Lay and biomedical understandings of musculoskeletal disorders. In: M. Elston (ed.) The Sociology of Medical Science and Technology. Oxford: Blackwell, pp. 79-99.

Calnan, M. and Gabe, J. (eds.) (2009) The restratification of primary care in England? In: The New Sociology of the Health Service. London: Routledge, pp. 56-78.

Berg, M. (1997) Problems and promises of the protocol. Social Science and Medicine 44(8): 1081-1088.

Bourdieu, P. (2000 [1996]) Pascalian Meditations. Cambridge: Polity.

Byrne, D. (2004) Evidence-based - What constitutes valid evidence. In: A. Gray and S. Harrison (eds.) Governing Medicine. Berks: Open University Press, pp. 81-92.

Carman, T. (2008) Merleau-Ponty. London: Routledge.

Checkland, C., Harrison, S., McDonald, R., Grant, S., Campbell, S. and Guthrie, B. (2008) Biomedicine, holism and general medical practice: Responses to the 2004 general practitioner contract. Sociology of Health and Illness 30(5): 788-803.

Corbin, J. and Strauss, A. (1985) Managing chronic illness at home: Three lines of work. Qualitative Sociology 8(3): 224-247.

Crossley, N. (1995) Merleau-Ponty, the elusive body and carnal sociology. Body and Society 1(1): 43-63.

Crossley, N. (1996) Body-subject/body-power: Agency, inscription and control in Foucault and Merleau-Ponty. Body and Society 2(2): 99-116.

Crossley, N. (2001) The Social Body. London: Routledge.

Department of Health. (2005) Self Care - A Real Choice. London: DH.

Department of Health. (2008) Raising the Profile of Long Term Conditions Care. Leeds, UK: DH.

Dreyfus, H. (1991) Being-in-the-World. Massachusetts: MIT Press.

Howson, A. and Inglis, D. (2001) The body in sociology: Tensions inside and outside sociological thought. The Sociological Review 49(3): 297-317.

Hughes, B. and Paterson, K. (1997) The Social model of disability and the disappearing body: Towards a sociology of impairment. Disability and Society 12(3): 325-340.

Jackson, M. (ed.) (1996) Things as They Are: New Directions in Phenomenological Anthropology. Bloomington, IN: Indiana University Press.

Kelly, M.P. and Field, D. (1996) Medical sociology, chronic illness and the body. Sociology of Health and Illness 18(2): 241-257.

Leder, D. (1990) The Absent Body. Chicago, IL: University of Chicago Press. 
Merleau-Ponty, M. (2002 [1962]) Phenomenology of Perception. London: Routledge.

Mol, A. and Law, J. (2004) Embodied action, enacted bodies: The example of hypoglycaemia. Body and Society 10(2-3): 43-62.

Prior, L. (2003) Belief, knowledge and expertise: The emergence of the lay expert in medical sociology. Sociology of Health and Illness 25(3): 41-57.

Rhodes, P., Langdon, M., Rowley, E., Wright, J. and Small, N. (2006) What does the use of a computerized checklist mean for patient-centred care? The example of a routine diabetes review. Qualitative Health Research 16(3): 353-376.

Rogers, A. (2009) Advancing the expert patient? Primary Health Care Research and Development 10: $167-176$.

Sullivan, M. (1986) In what sense is contemporary medicine dualistic? Culture, medicine and psychiatry 10: 331-350.

Toombs, S.K. (1992) The Meaning of Illness. Dordrecht, The Netherlands: Kluwer.

Turner, B. (2001) Disability and the sociology of the body. In: G.L. Albrecht, K.D. Seelman and M. Bury (eds.) Handbook of Disability Studies. Thousand Oaks, CA: Sage, pp. 252-266.

Smith, D. (1974) Women's perspective as a radical critique of sociology. Sociological Inquiry 44(1): 7-13.

Wanless, D. (2002) Securing Our Future Health. London: HM Treasury.

Williams, S.J. (1996) Medical Sociology, chronic illness and the body. Sociology of Health and Illness 18(5): 699-709.

Williams, S.J. (2006) Medical sociology and the biological body: Where are we now and where do we go from here? Health: An Interdisciplinary Journal 10(1): 5-30.

Williams, S.J. and Bendelow, G. (1998) The Lived Body. London: Routledge.

Young, A. (1981) When rational men fall sick: An inquiry into some assumptions made by medical anthropologists. Culture, Medicine and Psychiatry 5(4): 317-335.

Young, I.M. (2005) On Female Body Experience. Oxford: Oxford University Press. 